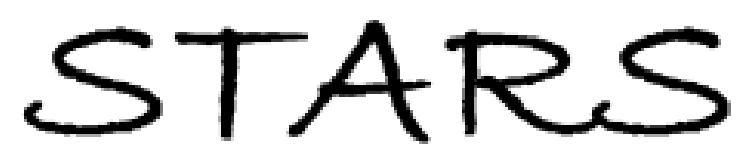

University of Central Florida

STARS

$1-1-2014$

\title{
Molecular-dynamics calculation of the vacancy heat of transport
}

\author{
Patrick K. Schelling \\ University of Central Florida \\ Jacques Ernotte \\ University of Central Florida \\ Lalit Schokeen \\ University of Central Florida \\ J. Woods Halley \\ William C. Tucker \\ University of Central Florida
}

Find similar works at: https://stars.library.ucf.edu/facultybib2010

University of Central Florida Libraries http://library.ucf.edu

This Article is brought to you for free and open access by the Faculty Bibliography at STARS. It has been accepted for inclusion in Faculty Bibliography 2010 s by an authorized administrator of STARS. For more information, please contactSTARS@ucf.edu.

\section{Recommended Citation}

Schelling, Patrick K.; Ernotte, Jacques; Schokeen, Lalit; Halley, J. Woods; and Tucker, William C., "Molecular-dynamics calculation of the vacancy heat of transport" (2014). Faculty Bibliography 2010 s. 6056.

https://stars.library.ucf.edu/facultybib2010/6056

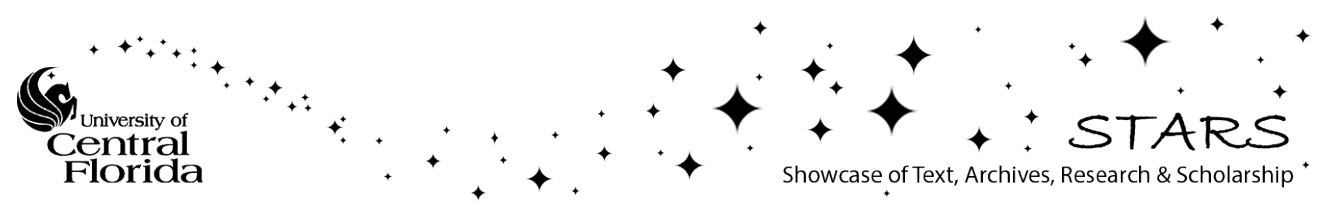




\section{Molecular-dynamics calculation of the vacancy heat of transport}

Cite as: J. Appl. Phys. 116, 023506 (2014); https://doi.org/10.1063/1.4886577

Submitted: 07 May 2014 . Accepted: 21 June 2014 . Published Online: 09 July 2014

Patrick K. Schelling, Jacques Ernotte, Lalit Shokeen, J. Woods Halley, and William C. Tucker
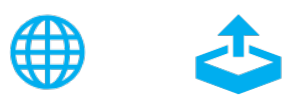

\section{ARTICLES YOU MAY BE INTERESTED IN}

Atomic-scale simulation of the thermodiffusion of hydrogen in palladium

Journal of Applied Physics 114, 063509 (2013); https://doi.org/10.1063/1.4816961

Analysis of simulation methodology for calculation of the heat of transport for vacancy thermodiffusion

Journal of Applied Physics 116, 023504 (2014); https://doi.org/10.1063/1.4887121

An empirical potential for silicon under conditions of strong electronic excitation

Applied Physics Letters 97, 151907 (2010); https://doi.org/10.1063/1.3499296

\section{Applied Physics Reviews} Now accepting original research

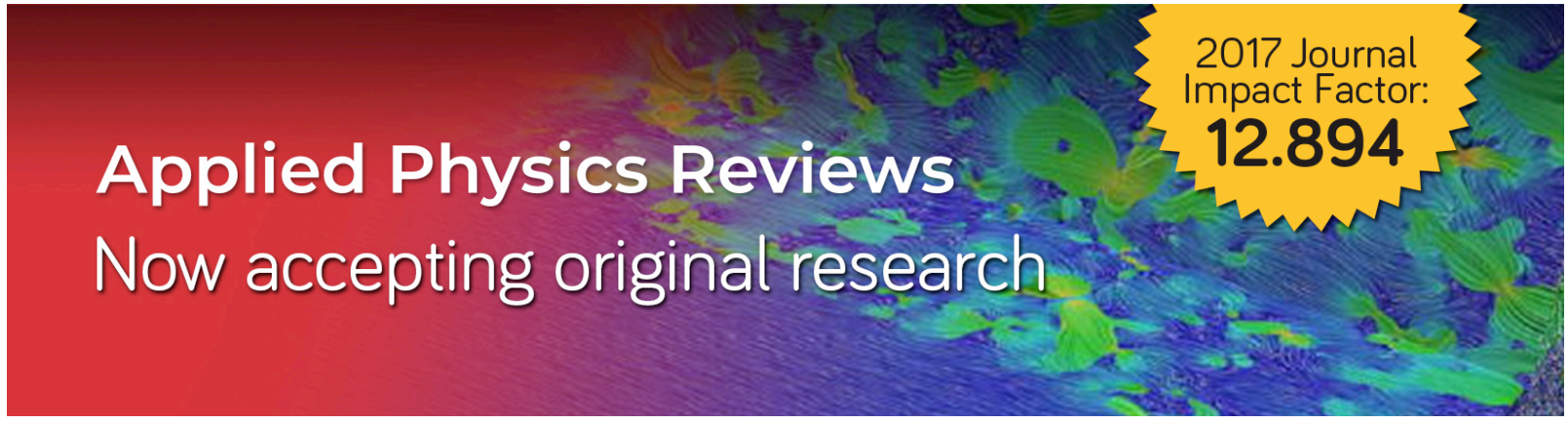




\title{
Molecular-dynamics calculation of the vacancy heat of transport
}

\author{
Patrick K. Schelling, ${ }^{1, \text { a) }}$ Jacques Ernotte, ${ }^{1, a)}$ Lalit Shokeen, ${ }^{1, a)}$ J. Woods Halley, ${ }^{2, b)}$ \\ and William C. Tucker ${ }^{1, a}$ \\ ${ }^{1}$ Advanced Material Processing and Analysis Center and Department of Physics, University of Central \\ Florida, 4000 Central Florida Blvd., Orlando, Florida 32816, USA \\ ${ }^{2}$ Department of Physics, University of Minnesota, 116 Church Street SE, Minneapolis, Minnesota 555455, \\ $U S A$
}

(Received 7 May 2014; accepted 21 June 2014; published online 9 July 2014)

\begin{abstract}
We apply the recently developed constrained-dynamics method to elucidate the thermodiffusion of vacancies in a single-component material. The derivation and assumptions used in the method are clearly explained. Next, the method is applied to compute the reduced heat of transport $Q_{v}^{*}-h_{f v}$ for vacancies in a single-component material. Results from simulations using three different Morse potentials, with one providing an approximate description of $\mathrm{Au}$, and an embedded-atom model potential for $\mathrm{Ni}$ are presented. It is found that the reduced heat of transport $Q_{v}^{*}-h_{f v}$ may take either positive or negative values depending on the potential parameters and exhibits some dependence on temperature. It is also found that $Q_{v}^{*}-h_{f v}$ may be correlated with the activation entropy. The results are discussed in comparison with experimental and previous simulation results. (C) 2014 AIP Publishing LLC. [http://dx.doi.org/10.1063/1.4886577]
\end{abstract}

\section{INTRODUCTION}

We have recently introduced a new method, termed constrained-dynamics, for computation of the heat of transport of vacancies and interstitial solutes. ${ }^{1,2}$ The method, which is quite different from earlier approaches, ${ }^{3-9}$ was shown to give satisfactory agreement when compared to Green-Kubo calculations, ${ }^{2}$ and generally reasonable values for the heat of transport in comparison to experiment. ${ }^{1}$ In Ref. 1, the central equation of the constrained-dynamics approach, in this case for computation of the reduced heat of transport for vacancies, $Q_{v}^{*}-h_{f v}$, was found to be

$$
Q_{v}^{*}-h_{f v}=W_{1 \rightarrow 2} \frac{T}{\Delta T},
$$

where $W_{1 \rightarrow 2}$ is the work done by the constraint force $F_{c}$ to move the constrained atom from $z_{1}$ to $z_{2}$, where $z_{1}$ and $z_{2}$ represent the positions of the local minima of the integrated work $W(z)$, given by

$$
W(z)=\int_{z_{1}}^{z}\left\langle F_{c}\left(z^{\prime}\right)\right\rangle d z^{\prime} .
$$

Hence, the work in Eq. (1) is given by $W_{1 \rightarrow 2}=W\left(z_{2}\right)$. The quantity $\left\langle F_{c}\left(z^{\prime}\right)\right\rangle$ is the time-average of the force required to hold the atom at $z^{\prime}$. Generally, the atom is displaced along the direction of the temperature gradient $\vec{\nabla} T$, and the temperature difference $\Delta T$ in Eq. (1) is found by integrating the temperature gradient along the path of the constrained atom. Specifically, $\Delta T=T_{1}-T_{2}$, where $T_{1}$ is the local temperature at $z_{1}$, and $T_{2}$ is the local temperature at $z_{2}$.

Before constrained-dynamics can become a standard approach, it is important to place the method on a more solid

\footnotetext{
a)Phone: (1)-407-882-1016. Fax: (1)-407-823-5112.

${ }^{b)}$ Phone/Fax: (1)-612-624-0395.
}

theoretical foundation. In Ref. 2, the predicted value for the heat of transport of hydrogen atoms in a Pd lattice was computed using the constrained-dynamics method and found to be in essentially exact agreement with standard Green-Kubo techniques. This provides some support for the constraineddynamics approach. While this is encouraging, the limited study in Ref. 2 probably is not sufficient to validate the approach. One challenge is that in the case of vacancymediated thermodiffusion, Green-Kubo methods are not practical and it is difficult to directly validate the constrained-dynamics approach. More broadly, there are many problems where the diffusion rate is too slow to directly apply Green-Kubo techniques to validate predictions using the constrained-dynamics approach. Yet it is precisely these problems that constrained-dynamics was developed to address.

In this article, we examine the assumptions made in the derivation of Eq. (1). In particular, we present a more thorough derivation meant to address questions that have arisen after publication of Ref. 1. The method is then used to compute the heat of transport of vacancies in model fcc metals using Morse potentials and an embedded-atom model (EAM) potential for Ni. It is shown that the computed values for the reduced heat of transport $Q_{v}^{*}-h_{f v}$ vary in a systematic manner with the parameters of the potentials, and moreover, appear to be correlated with the activation entropy.

\section{THEORETICAL BACKGROUND}

We consider here specifically the thermodiffusion of vacancies in a single-component material. Here, we present a more thorough discussion than Ref. 1, with the objective of clarifying the relation of the quantities in Eq. (1) with the quantities calculated in the constrained molecular dynamics calculation which we carry out to determine them. We will also demonstrate that the method obeys the laws of 
thermodynamics applied to irreversible processes. In the following, we omit vector notation for simplicity. The vacancy and energy current densities, $J_{v}$ and $J_{q}$, are given by the expressions first presented in Ref. 1

$$
\begin{aligned}
& J_{v}=L_{v v} X_{v}, \\
& J_{q}=L_{q q} X_{q},
\end{aligned}
$$

where $X_{v}$ and $X_{q}$ represent the driving forces for mass and heat transport. The driving forces are given by

$$
\begin{gathered}
X_{v}=-F_{\text {ext }}-T \nabla\left(\frac{\mu_{v}}{T}\right)+Q_{v}^{*}\left(-\frac{\nabla T}{T}\right) \\
=k_{B} T\left(-\frac{\nabla c_{v}}{c_{v}}\right)+\left(Q_{v}^{*}-h_{f v}\right)\left(-\frac{\nabla T}{T}\right), \\
X_{q}=-\frac{\nabla T}{T},
\end{gathered}
$$

where $F_{\text {ext }}$ is a (coarse-grained) external force, $c_{v}$ is the vacancy concentration, $Q_{v}^{*}$ is the vacancy heat of transport, and $h_{f v}$ is the enthalpy of formation for vacancies. The negative sign for the external force is to indicate that forces are applied to atoms rather than directly to vacancies. In Ref. 10, a thorough discussion of the phenomenological transport equations can be found. With appropriate definitions of the transport coefficients the equation for the vacancy current $J_{v}$ can be rewritten, taking account of an external force applied to the atoms as

$$
J_{v}=-D_{v} \nabla n_{v}+\left(\frac{D_{v}}{k_{B} T}\right) n_{v}\left[-F_{e x t}+\left(Q_{v}^{*}-h_{f v}\right)\left(-\frac{\nabla T}{T}\right)\right],
$$

where $D_{v}=L_{v v} k_{B} T / n_{v}$. This equation implicitly defines the reduced heat of transport $Q_{v}^{*}-h_{f v} \equiv Q_{v}^{\prime *}$ which we wish to compute. For our purposes, it is important to emphasize that the relations Eqs. (3)-(7) refer to quantities averaged in space and time over scales long compared to the typical dynamic time scales in the system and similarly over spatial distances at least as large as the interatomic distances in the system of interest. In the case at hand, we are concerned with the transport of vacancies which takes place via hopping of atoms (in the direction opposite to that of the vacancy current) over distances of the order of the lattice spacing. These hops are rare events which take place quickly (less than $1 \mathrm{ps)} \mathrm{separated} \mathrm{by} \mathrm{long} \mathrm{residence} \mathrm{times} \mathrm{(nanosec-}$ onds or more depending on the hopping barrier and temperature). The time scales on which the macroscopic Eqs. (3)-(7) apply are the latter times. Details of the hopping dynamics have been averaged away in the coarse graining. Similarly, spatial details of the path followed during the hop have been integrated out in the spatial coarse graining, and the smallest relevant spatial scale is the distance $\Delta z$ along the thermal gradient between metastable positions of the vacancy. Thus when relating microscopic quantities computed in molecular dynamics simulations to the variables in these equations, we can regard the thermal gradient as $\Delta T / \Delta z$ in which $\Delta z$ is the distance between metastable positions of the vacancies along the direction of the thermal gradient and $\Delta T$ is the corresponding difference in the local temperature.
In the molecular dynamics calculations used to implement Eq. (7), a time-varying constraint force $F_{c}(z)$ is applied to the atom which would otherwise undergo a hop, holding it at a fixed position $z$ along the path between local minima. Effectively, the Hamiltonian of the constrained system for pair-wise interactions with potential energy $\phi(r)$ is

$$
H(z)=\sum_{\mu=1}^{2} \frac{p_{1, \mu}^{2}}{2 m}+\sum_{i=2}^{N} \frac{\vec{p}_{i} \cdot \vec{p}_{i}}{2 m}+\sum_{j=2}^{N} \phi\left(r_{1 j}\right)+\sum_{i=j+1}^{N} \sum_{j=2}^{N-1} \phi\left(r_{i j}\right) .
$$

Here, the constrained atom corresponds to $i=1$, and the index $\mu$ represents the Cartesian components. The position vector of atom $i=1$ is then $\vec{r}_{1}=x_{1} \hat{i}+y_{1} \hat{j}+z \hat{k}$. Hence the $\mathrm{z}$-coordinate of the hopping atom is constrained and given by $z_{1}=z$. The z-component of the momentum of the hopping atom is zero, $p_{1,3}=0$. Thus the simulation does not simulate the dynamics of the hop but constrains the hopping atom to move adiabatically from one stable minimum to the next in the presence of a thermal gradient imposed by the boundary conditions on the simulation. The average force imposed over the passage from one stable minimum to the next is

$$
F_{e x t}=\frac{1}{z_{2}-z_{1}} \int_{z_{1}}^{z_{2}}\left\langle F_{c}\left(z^{\prime}\right)\right\rangle d z^{\prime}=\frac{W_{1 \rightarrow 2}}{z_{2}-z_{1}},
$$

where the brackets on $\left\langle F_{c}\left(z^{\prime}\right)\right\rangle$ denote a time average in the molecular-dynamics simulation, assumed to be equivalent by the usual ergodicity assumption to a thermal average in an ensemble representing the local thermodynamic equilibrium. Thus $F_{\text {ext }}$ is a coarse-grained force, appropriate for inclusion in the macroscopic description of the constrained calculation in Eq. (7). During this molecular dynamics calculation, there is only one vacancy and it is not allowed to flow. Thus there is no spatial gradient in the density and no vacancy current. In these conditions, the macroscopic Eq. (7) becomes

$$
0=\left(\frac{D_{v}}{k_{B} T}\right) n_{v}\left[-F_{e x t}+Q_{v}^{* \prime}\left(-\frac{\nabla T}{T}\right)\right] .
$$

For the temperature gradient, we take $\nabla T=\frac{T_{1}-T_{2}}{z_{1}-z_{2}}$, and then obtain

$$
F_{e x t}=-\left(\frac{Q_{v}^{* \prime}}{T}\right)\left(\frac{T_{1}-T_{2}}{z_{1}-z_{2}}\right) .
$$

Inserting Eq. (9) for $F_{e x t}$, we recover the central equation of the method, Eq. (1). One can regard the integral in Eq. (9) as the work required to slowly return the atom from $z_{1}$ to $z_{2}$ after a hop as described in the Appendix, where we also show that there is no violation of the second law in the processes that are involved.

In Sec. III, specific technical details are given about the constrained-dynamics method. These were reported in Refs. 1 and 2 but are reproduced here for completeness. Details of the particular empirical potentials used are also given.

\section{SIMULATION APPROACH}

We report here simulation results using three different Morse-type potentials and an EAM potential for Ni. The Morse potentials take the form 


$$
V(r)=\epsilon\left[e^{-2 \alpha\left(r-r_{0}\right)}-2 e^{\alpha\left(r-r_{0}\right)}\right] .
$$

In the MD simulations using Morse potentials we express energies in units of $\epsilon$, masses in units of the mass $m$ of the atom simulated, lengths in units of $r_{0}$, and time in units of $r_{0} \sqrt{m / \epsilon}$. In these units, we chose a time step of $\Delta t^{*}=0.001$. We chose three different values of $\alpha r_{0}$. In particular, we consider $\alpha r_{0}=4.55, \alpha r_{0}=6.0$, and $\alpha r_{0}=8.0$. The Morse potential has a minimum at $r-r_{0}=0$, where the value is $V$ $\left(r=r_{0}\right)=-\epsilon$. The cutoff for the interactions was taken to be $r_{\text {cut }}=3.0 r_{0}$, which in each case is somewhat greater than twice the lattice parameter at zero reduced pressure $p^{*}=0$. The parameterization $\alpha r_{0}=4.55$ was used in the Cherns potential $^{12}$ for Au. The Cherns potential for Au was implemented in calculations of the vacancy heat of transport as reported in Refs. 7-9.

Using the Morse potentials described above, we performed simulations at the reduced temperature $T^{*}=$ $\frac{k_{B} T}{\varepsilon}=0.15$ and several different fixed volumes. At each volume, the time-averaged pressure was computed. The reduced pressure, $p^{*}$, as a function of reduced volume $V^{*}$ is shown in Fig. 1. The results in Fig. 1 were obtained from $10^{4} \mathrm{MD}$ time steps without including the first $10^{3}$ steps of equilibration. We fit the data in Fig. 1 to the Birch-Murnaghan equation of state

$$
\begin{aligned}
p^{*}\left(V^{*}\right)= & \frac{3 B_{0}^{*}}{2}\left[\left(\frac{V_{0}^{*}}{V^{*}}\right)^{7 / 3}-\left(\frac{V_{0}^{*}}{V^{*}}\right)^{5 / 3}\right] \\
& \times\left[1+\frac{3}{4}\left(B_{0}^{\prime}-4\right)\left[\left(\frac{V_{0}^{*}}{V^{*}}\right)^{2 / 3}-1\right]\right],
\end{aligned}
$$

where $B_{0}^{*}$ is the reduced bulk modulus at zero pressure, $B_{0}^{\prime}$ is the pressure derivative of the bulk modulus, $V^{*}$ is the reduced system volume per atom, and $V_{0}^{*}$ is the reduced volume per atom for $p^{*}=0$. The results are shown in Table I in reduced units.

To produce results that are suitable for comparison with real materials, we chose values for $r_{0}$ and $\epsilon$. We define in each simulation $r_{0}=3.035 \AA$, and $\epsilon=0.5838 \mathrm{eV}$. With these

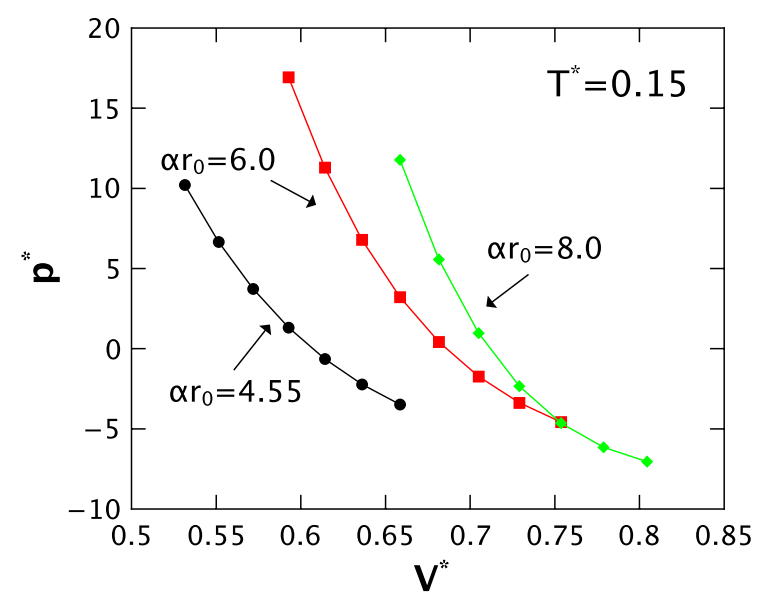

FIG. 1. Computed values of the reduced pressure $p^{*}$ as a function of the reduced volume per atom $V^{*}$ at a reduced temperature $T^{*}=0.15$. The results were determined for potential parameters $\alpha r_{0}=4.55$ (black circles), $\alpha r_{0}=6.0$ (red squares), and $\alpha r_{0}=8.0$ (green diamonds).
TABLE I. Computed materials parameters in reduced units for Morse potentials with different values for $\alpha r_{0}$.

\begin{tabular}{lccc}
\hline \hline Property & $\alpha r_{0}=4.55$ & $\alpha r_{0}=6.0$ & $\alpha r_{0}=8.0$ \\
\hline$B_{0}^{*}$ & 53.8 & 68.7 & 106.1 \\
$V_{0}^{*}$ & 0.607 & 0.685 & 0.711 \\
$B_{0}^{\prime}$ & 5.45 & 7.48 & 9.31 \\
\hline \hline
\end{tabular}

choices, we obtain $a_{0}=4.08 \AA$ and $B_{0}=180 \mathrm{GPa}$ at a reduced temperature $T^{*}=0.15$ and pressure $p^{*}=0$ for the potential with $\alpha r_{0}=4.55$. These values correspond to the experimentally measured properties of $\mathrm{Au}$ at room temperature. The resulting materials properties for each potential, including the value of $\alpha$ that characterizes the three potentials, are reported in Table II. We also compute the cohesive energy predicted by each potential using the volume predicted for $p^{*}=0$ and $T^{*}=0.15$. In comparison to the experimental value $E_{c o h}=3.81 \mathrm{eV}$ for $\mathrm{Au}$, we see that the potential with $\alpha r_{0}=4.55$ predicts a significantly larger value. Therefore, the potential with $\alpha r_{0}=4.55$, while representative of $\mathrm{Au}$, is not rigorously fit. The potentials with parameterization $\alpha r_{0}=6.0$ and $\alpha r_{0}=8.0$ do not fit any particular materials system but are included here to determine how $Q_{v}^{*}-h_{f v}$ might systematically depend or be correlated with other materials properties. From the value $\epsilon$ and the reduced temperature $T^{*}=0.15$, we obtain the actual simulation temperature $T=1016 \mathrm{~K}$. Defining the atomic mass to be equal to that of $\mathrm{Au}$, we find that the reduced MD time step of $\Delta t^{*}=0.001$ corresponds to an actual value of $\Delta t=0.568 \mathrm{fs}$, which is sufficient to ensure good energy conservation.

The EAM potential for $\mathrm{Ni}$ used here is described in Refs. 13 and 14. The potential was optimized for the Ni-Al system, but the empirical potential used here was specifically developed in Ref. 13 for pure Ni. Included in the fitting database were the lattice parameter, cohesive energy, and elastic constants. Reasonable phonon dispersion curves were reported in Ref. 13, although zone-edge phonon modes appear to be over predicted by $\sim 10 \%-20 \%$ in comparison to experiment. In addition, vacancy formation and migration energies, which are quite relevant for the heat of transport, were found to be within a few percent of experimental values. For example, the vacancy formation energy was computed to be $1.19 \mathrm{eV}$, which is quite close to the experimental value $1.30 \mathrm{eV} .^{13}$ These points will be revisited in Sec. IV.

The simulation supercell was a rectangular prism. We define a Cartesian coordinate system coincident with the vectors that define the supercell. The $\mathrm{x}^{-}, \mathrm{y}^{-}$, and $\mathrm{z}-$ directions

TABLE II. Potential parameters and resulting material properties computed at $T^{*}=0.15$.

\begin{tabular}{lccc}
\hline \hline Parameter & $\alpha r_{0}=4.55$ & $\alpha r_{0}=6.0$ & $\alpha r_{0}=8.0$ \\
\hline$\alpha\left(\AA^{-1}\right)$ & 1.4992 & 1.9769 & 2.6359 \\
$\epsilon(\mathrm{eV})$ & 0.5838 & 0.5838 & 0.5838 \\
$r_{0}(\AA)$ & 3.0350 & 3.0350 & 3.0350 \\
$B_{0}(\mathrm{GPa})$ & 180.0 & 229.9 & 355.0 \\
$V_{0}\left(\AA^{3}\right)$ & 16.98 & 19.19 & 20.02 \\
$a_{0}(\AA)$ & 4.08 & 4.25 & 4.31 \\
$E_{c o h}(\mathrm{eV})$ & 4.85 & 4.01 & 3.66 \\
\hline \hline
\end{tabular}


were coincident with the [11̄0], [001], and [110] crystal directions, respectively. With this supercell, it is convenient to define a two-atom unit cell. For the Morse potentials, the supercell used consisted of $10 \times 7 \times 28$ unit cells with a total of $N=3920$ fcc lattice sites. For simulations using the EAM Ni potential, a slightly smaller supercell was used due to the fact that the EAM potentials were more computationally intense. Specifically, for EAM Ni simulations, a supercell with $8 \times 6 \times 28$ unit cells and a total of $N=2688 \mathrm{fcc}$ lattice sites was used. We introduced a single vacancy near the center of the simulation cell and constrained one of the atoms at various points corresponding to a vacancy hop along the [110] direction. Specifically, at the beginning of each MD simulation, the hopping atom was displaced by the desired amount from the equilibrium position $z_{1}$. In addition, its initial velocity component along [110] was set to zero. During the MD simulation, the forces were computed on all the atoms, including the force $\vec{F}$ felt by the hopping atom due to the interactions with the neighboring atoms in the simulation cell. Next, the component of $\vec{F}$ along the [110] direction, which is coincident with the $z$-axis in the Cartesian system defined above, was computed from $\vec{F} \cdot \hat{k}$. The constraint force $\vec{F}_{c}$ is then given by

$$
\vec{F}_{c}=-(\vec{F} \cdot \hat{k}) \hat{k} .
$$

This constraint force was then added to the force acting on the hopping atom, so that the resulting force $\vec{F}^{\prime}$ was

$$
\vec{F}^{\prime}=\vec{F}+\vec{F}_{c} .
$$

The hopping atom followed the appropriate dynamics corresponding to $\vec{F}^{\prime}$. Because $\vec{F}^{\prime}$ has no component along the [110] direction, there was no motion in that direction. However, the hopping atom was not constrained in the directions perpendicular to [110], so that effectively all possible trajectories from $z_{1}$ to $z_{2}$ are explored. Finally, so that the center of mass of the system does not move when $\vec{F}_{c}$ was applied to the constrained atom, and equal but opposite force was applied to the other atoms in the system. In particular, in a system with $N$ lattice sites and $N-1$ atoms, the force on the hopping atom is given by Eq. (15), and the forces on the $N-2$ other atoms were given by

$$
\vec{F}_{k}^{\prime}=\vec{F}_{k}-\frac{\vec{F}_{c}}{N-2}
$$

where $\vec{F}_{k}^{\prime}$ is the resulting force on atom $k$, and $\vec{F}_{k}$ is the force on atom $k$ determined from the empirical potential. Thus, the additional force due to the constraint is added uniformly to the $N-2$ atoms, and apart from the hopping atom, the other $N-2$ atoms followed the dynamics suggested by Eq. (16). It might also be added that dynamics that result from Eq. (15) for the hopping atom and Eq. (16) for the other $N-2$ atoms allow the system to relax fully when the atom is placed in between $z_{1}$ and $z_{2}$. In general, placing the hopping atom near the transition state results in large forces which cause a local distortion in the lattice.

The simulation details for the Morse potentials and the Ni EAM potentials were somewhat different. In the case of the Morse potentials, for each constrained position along the transition path, we first simulated for $10^{6} \mathrm{MD}$ steps at constant reduced temperature $T^{*}=0.15$. After $10^{5} \mathrm{MD}$ steps to reach equilibrium, statistics for the average energy and constraint force are accumulated. Next, we use the final positions and velocities of the atoms as a starting point for simulation in the presence of a temperature gradient. The basic approach is what was previously reported in Ref. 1 . In particular, we designate two regions of thickness $\delta=0.05 L_{z}$, where $L_{z}$ is the length of the simulation cell along the $\mathrm{z}$-direction. The midpoints of the two regions are separated by a distance $L_{z} / 2$, and the vacancy is approximately equidistant from the regions. In these two regions, velocity rescaling following the algorithm developed in Ref. 15 is used to create hot and cold reservoirs. At each MD step, an energy $\Delta E=0.05 \epsilon$ is added to the hot region, and the same amount of energy $\Delta E$ is removed from the cold region. After turning on the velocity rescaling, the simulation is run for $2 \times 10^{6}$ MD steps for each location of the constrained atom. The first $10^{5} \mathrm{MD}$ steps are used to achieve steady-state with the imposed thermal current. After achieving steady state, the constraint force is time averaged over the remaining $1.9 \times 10^{6}$ MD steps.

For simulations using the EAM potential, the MD time step was taken to be $\Delta t=0.358 \mathrm{fs}$. The system was first equilibrated at constant temperature $T=450 \mathrm{~K}$ for $2 \times 10^{5} \mathrm{MD}$ steps. After equilibration, the temperature gradient is added using the same approach as described above, including the thickness $\delta=0.05 L_{z}$ of the hot and cold reservoirs. At each MD step, an energy $0.01 \mathrm{eV}$ is added/removed from the hot/cold reservoirs. The simulation with the applied heat current included $2 \times 10^{6} \mathrm{MD}$ steps. After $10^{5} \mathrm{MD}$ steps to reach a steady-state temperature gradient, the statistics for the average constraint force were accumulated over the remaining $1.9 \times 10^{6} \mathrm{MD}$ steps.

The integrated work $W(z)$ performed by the constraint force was computed as a function of $z$ using Eq. (2). In equilibrium, the integrated work $W(z)$ is related to the freeenergy profile. When a temperature gradient is applied, using the approach described above, the integrated work from the starting position $z_{1}$ to the final local minimum $z_{2}$ is determined, with $W_{1 \rightarrow 2}=W\left(z_{2}\right)$. This quantity is used in Eq. (1) to compute $Q_{v}^{*}-h_{f v}$.

It should be remarked that a similar procedure has been applied in Ref. 11 to compute the thermodiffusion of nanoparticles in a solvent. Specifically, in Ref. 11, the nanoparticle was connected to a spring which provides, on average, a force that opposes the thermodiffusion driving force due to an applied heat current. By computing the average spring force required to hold the nanoparticle in place, the thermodiffusion coefficient was determined. We believe that this approach is similar to the constrained-dynamics approach in that the external force required to balance the driving force for thermodiffusion is computed. However, the approach in Ref. 11 cannot be applied to the problem of vacancy thermodiffusion due to the very long time scales associated with vacancy hopping. However, we do think that the constrained-dynamics approach could be applied to the problems studied in Ref. 11. 


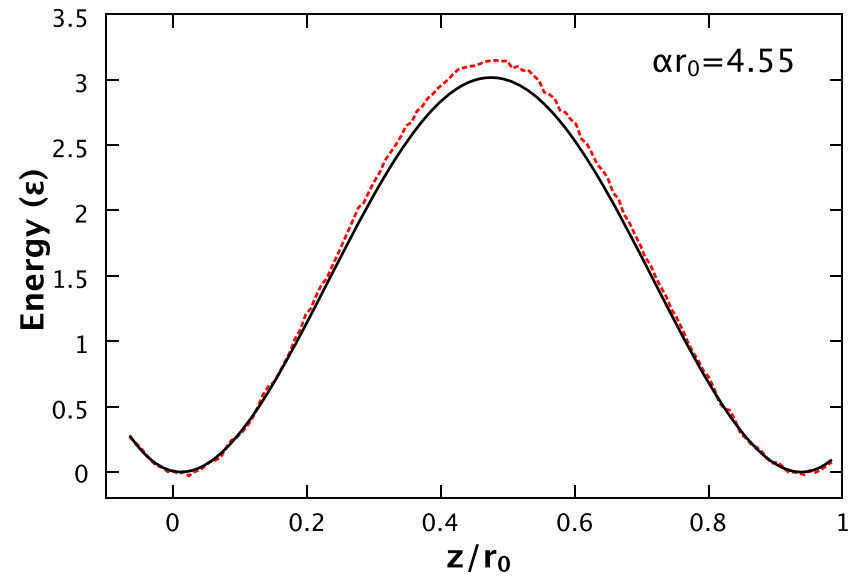

FIG. 2. The internal energy $\Delta U(z)$ (dashed line) and free-energy $\Delta A(z)$ (solid line) in units of $\epsilon$ as a function of the coordinate $z$ of the constrained atom for $\alpha r_{0}=4.55$. The results were computed in isothermal conditions $\nabla T^{*}=0$ with $T^{*}=0.15$.

\section{RESULTS}

We begin by presenting the results obtained using the Morse potentials. In isothermal conditions $\nabla T^{*}=0$, integration of the average constraint force is related to the potential of mean force, or, alternately, the free-energy profile. The free energy difference $\Delta A(z)=A(z)-A\left(z_{1}\right)$ is found from integrating the time-averaged constraint force $\left\langle F_{c}(z)\right\rangle$. Hence, $\Delta A(z)=W(z)$ with $W(z)$ computed using Eq. (2). We define the energy difference $\Delta U(z)=U(z)-U\left(z_{1}\right)$. In Fig. 2, we show the computed profiles $\Delta A(z)$ and $\Delta U(z)$ for $\alpha r_{0}=4.55$. The transition state occurs exactly in the middle of the path at $z / r_{0} \approx 0.475$. We find the activation free energy $\Delta A_{a}=(3.017 \pm 0.002) \epsilon$. For the activation energy, we find $\Delta U_{a}=(3.15 \pm 0.02) \epsilon$. The magnitude of the numerical error in the calculation of $\Delta A_{a}$ and $\Delta U_{a}$ for $\alpha r_{0}=6.0$ and $\alpha r_{0}=8.0$ is similar. In Table III, we show the results for $\Delta A_{a}$ and $\Delta U_{a}$ computed for each value of $\alpha r_{0}$ simulated in isothermal conditions with $T^{*}=0.15$. As the value $\alpha r_{0}$ increases, both $\Delta A_{a}$ and $\Delta U_{a}$ increase. This is easily understood. In particular, as $\alpha r_{0}$ increases, the interatomic potential becomes increasingly short-ranged, and as a result both $\Delta A(z)$ and $\Delta U(z)$ exhibit larger increases as the transition state is approached. We also note from Table III that for $\alpha r_{0}=4.55$ and $\alpha r_{0}=6.0, \Delta U_{a}>\Delta A_{a}$, whereas for $\alpha r_{0}=8.0, \Delta U_{a}<\Delta A_{a}$. This indicates a change in sign of the activation entropy $\Delta S_{a}$ as $\alpha r_{0}$ increases.
In Fig. 3, we plot the entropy difference $\Delta S(z)$ defined by

$$
\Delta S(z)=\frac{\Delta U(z)-\Delta A(z)}{T}
$$

for the case $\alpha r_{0}=4.55$. We find generally that $\Delta S(z)>0$, although noise in the data makes it impossible to conclude that $\Delta S(z)$ is positive for all values of $z$. This is opposite the behavior found in Ref. 1, where $\Delta S(z)$ was found to be negative. The noise in the plot of $\Delta S(z)$ is the result primarily of numerical fluctuations in the value of $\Delta U(z)$, since fluctuations throughout the entire system are involved in the computed value of $\Delta U(z)$. The entropy at the transition state, known as the activation entropy $\Delta S_{a}$, is positive and equal to $\Delta S_{a}=(0.85 \pm 0.15) k_{B}$. Measurement of $\Delta S_{a}$ is not straightforward, and different methods of calculation can yield different results. ${ }^{16}$ However, we are unaware of any argument that suggests that either $\Delta S_{a}<0$ or $\Delta S_{a}>0$ is not possible.

We find that the activation entropy $\Delta S_{a}$ depends sensitively on the choice of the parameter $\alpha r_{0}$. In Fig. 4, we show the computed profile $\Delta S(z)$ for $\alpha r_{0}=6.0$, and in Fig. 5, we show the same quantity for $\alpha r_{0}=8.0$. Comparison of Figs. 3-5 shows that $\Delta S_{a}$ gradually decreases (i.e., trends towards negative values) as $\alpha r_{0}$ increases. For example, in Fig. 4 for $\alpha r_{0}=6.0, \Delta S_{a}$ is positive, but significantly smaller than $\Delta S_{a}$ for $\alpha r_{0}=4.55$. Likewise, in Fig. 5, $\Delta S(z)$ appears to be negative across the entire region between $z_{1}$ and $z_{2}$. In Table III, the computed values of $\Delta S_{a}$ are given for each value of $\alpha r_{0}$ along with the associated numerical error. We will show below that the observed trend of decreasing $\Delta S_{a}$ with increasing $\alpha r_{0}$ appears to be closely linked with the computed value of $Q_{v}^{*}-h_{f v}$.

To determine the reduced heat of transport $Q_{v}^{*}-h_{f v}$, we followed the general approach developed in Ref. 1 and discussed thoroughly above. Specifically, the work $W_{1 \rightarrow 2}$ is the work done by the constraint force to drive the constrained atom from $z_{1}$ to $z_{2}$, where $z_{1}$ and $z_{2}$ represent the positions of the constrained atom that correspond to a local minima of the integrated work. The integrated work $W(z)$ is given by Eq. (2), with $W_{1 \rightarrow 2}=W\left(z_{2}\right)$. We take $z_{1}$ to the be the position of the local minimum nearest to the hot region, and $z_{2}$ be the position of the local minimum nearest to the cold region. To accurately determine $z_{1}, z_{2}$, and $W_{1 \rightarrow 2}$, we fit the integrated work $W(z)$ to a cubic polynomial in the vicinity of $z_{1}$ and $z_{2}$.

In Fig. 6, we show the integrated work $W(z)$ near $z=z_{2}$ along with the fits to a cubic polynomial for each value of

TABLE III. Computed quantities related to diffusion of a vacancy given in reduced units. The values in parentheses are the corresponding energies determined using $\epsilon=0.5838 \mathrm{eV}$. The values for the activation free energy $\Delta A_{a}$, energy $\Delta U_{a}$, and entropy $\Delta S_{a}$ are computed in thermal equilibrium at reduced temperature $T^{*}=0.15$. When a temperature gradient is present, there is a difference $\Delta T^{*}$ in the reduced temperature between the initial and final location of the diffusing atom. The values for the activation entropy $\Delta S_{a}$ are given in units of $k_{B}$. The temperature difference $\Delta T^{*}$ is also given in reduced units.

\begin{tabular}{|c|c|c|c|}
\hline Parameter & $\alpha r_{0}=4.55$ & $\alpha r_{0}=6.0$ & $\alpha r_{0}=8.0$ \\
\hline$\Delta A_{a}$ & $3.017 \pm 0.002(1.762)$ & $4.829 \pm 0.002(2.819)$ & $7.397 \pm 0.002(4.319)$ \\
\hline$\Delta U_{a}$ & $3.15 \pm 0.02(1.84)$ & $4.86 \pm 0.02(2.84)$ & $7.24 \pm 0.02(4.23)$ \\
\hline$\Delta S_{a}\left(k_{B}\right)$ & $0.85 \pm 0.15$ & $0.20 \pm 0.15$ & $-1.05 \pm 0.10$ \\
\hline$\Delta T^{*}$ & $0.0101 \pm 0.0020$ & $0.0072 \pm 0.0015$ & $0.0063 \pm 0.0014$ \\
\hline$W_{1 \rightarrow 2}$ & $-0.025 \pm 0.002(-0.013)$ & $-0.011 \pm 0.002(-0.006)$ & $0.004 \pm 0.002(0.002)$ \\
\hline$Q_{v}^{*}-h_{f v}$ & $-0.39 \pm 0.08(-0.22)$ & $-0.23 \pm 0.05(-0.13)$ & $0.09 \pm 0.01(0.06)$ \\
\hline
\end{tabular}




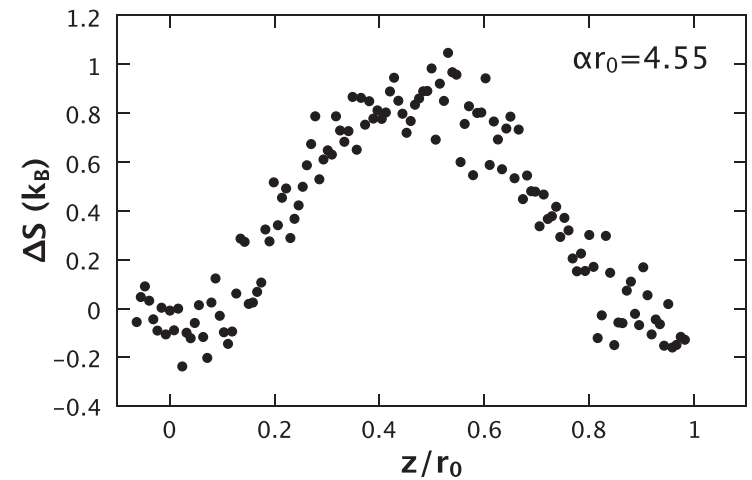

FIG. 3. Entropy change $\Delta S(z)$ (in units of $k_{B}$ ) as a function of the coordinate $z / r_{0}$ of the constrained atom for $\alpha r_{0}=4.55$. The simulation is performed in isothermal conditions $\nabla T^{*}=0$ with reduced temperature $T^{*}=0.15$.

$\alpha r_{0}$. The minimum of the fit curve is used to determine $z_{2}$ and $W_{1 \rightarrow 2}$. The position $z=z_{2}$ corresponds to the constrained atom in a region with slightly lower local temperature in comparison to the local temperature at $z=z_{1}$. The most important result of this paper is the systematic dependence of $W_{1 \rightarrow 2}$ on the potential parameter $\alpha r_{0}$. In particular, Fig. 6 shows that for $\alpha r_{0}=4.55, W_{1 \rightarrow 2}$ is strongly negative. For $\alpha r_{0}=6.0, W_{1 \rightarrow 2}$ is still negative but is much closer to zero. In Ref. $1, W_{1 \rightarrow 2}$ was also found to be negative and of very similar magnitude. In sharp contrast to these results, we found that for $\alpha r_{0}=8.0$, the value of $W_{1 \rightarrow 2}$ becomes positive.

To determine the value of $Q_{v}^{*}-h_{f v}$, we use Eq. (11). Hence, we need the computed temperature difference $\Delta T^{*}$, which is given by

$$
\Delta T^{*}=T^{*}\left(z_{1}\right)-T^{*}\left(z_{2}\right)=T_{1}-T_{2},
$$

which is found by fitting the computed temperature profile in the region near the vacancy as was previously done in Ref. 1. In Table III, we show the computed values of $W_{1 \rightarrow 2}$, $\Delta T^{*}$, and $Q_{v}^{*}-h_{f v}$. The numerical error for $W_{1 \rightarrow 2}, \Delta T^{*}$, and $Q_{v}^{*}-h_{f v}$ are also given in Table III. The primary source of error in $Q_{v}^{*}-h_{f v}$ is due to uncertainty about the value of $\Delta T^{*}$. We find that $Q_{v}^{*}-h_{f v}$ is large and negative for $\alpha r_{0}=4.55$ and trends towards positive values as $\alpha r_{0}$ increases. For $\alpha r_{0}=8.0, Q_{v}^{*}-h_{f v}$ is rather small but

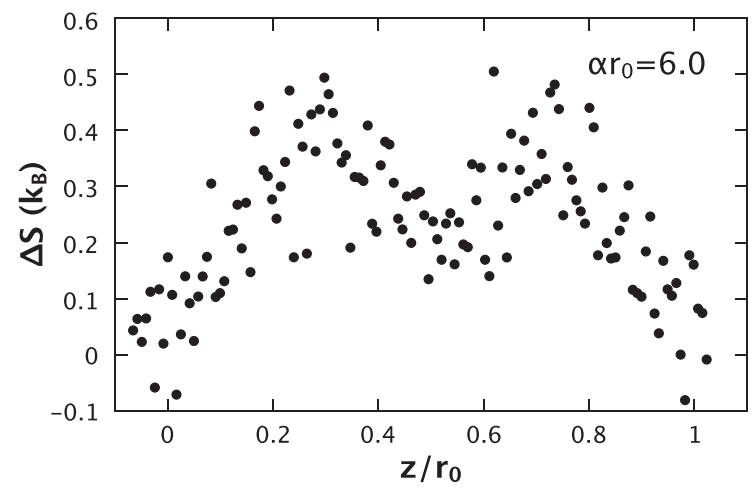

FIG. 4. Entropy change $\Delta S(z)$ (in units of $k_{B}$ ) as a function of the coordinate $z / r_{0}$ of the constrained atom for $\alpha r_{0}=6.0$. The simulation is performed in isothermal conditions $\nabla T^{*}=0$ with reduced temperature $T^{*}=0.15$.

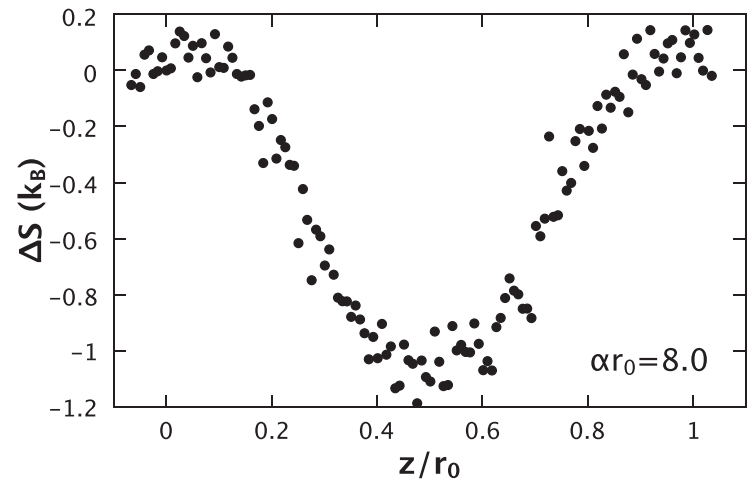

FIG. 5. Entropy change $\Delta S(z)$ (in units of $k_{B}$ ) as a function of the coordinate $z / r_{0}$ of the constrained atom for $\alpha r_{0}=8.0$. The simulation is performed in isothermal conditions $\nabla T^{*}=0$ with reduced temperature $T^{*}=0.15$.

nevertheless clearly positive. This indicates that the details of the empirical potential can result in a qualitative change in the nature of the driving force for thermodiffusion. In particular, when $Q_{v}^{*}-h_{f v}<0$, the tendency is for a vacancy to be driven towards the high-temperature region. By contrast, the fact that $Q_{v}^{*}-h_{f v}>0$ for $\alpha r_{0}=8.0$ indicates that in that case, vacancies will tend to be driven towards the lowtemperature region.

We next consider the possibility of temperature dependent values of $Q_{v}^{*}-h_{f v}$. In Ref. 8, the reported calculations suggest that $Q_{v}^{*}-h_{f v}$ exhibits strong temperature dependence. To explore the question of temperature dependence, we also simulated the case where $\alpha r_{0}=4.55$ for an average temperature $T^{*}=0.20$. The system volume was chosen to be the same as that of the system with $T^{*}=0.15$, which resulted in larger pressure due to the tendency of the system to expand at higher temperatures. In Fig. 7, we show the integrated work for $\alpha r_{0}=4.55$ for the two temperatures $T^{*}=0.15$ and $T^{*}=0.20$. We indeed observe a significant dependence on $T^{*}$. For $T^{*}=0.20$, we find $W_{1 \rightarrow 2}=(-0.031 \pm 0.002) \epsilon$, which results in a reduced heat of transport $Q_{v}^{*}-h_{f v}=(-0.59 \pm 0.12) \epsilon$.

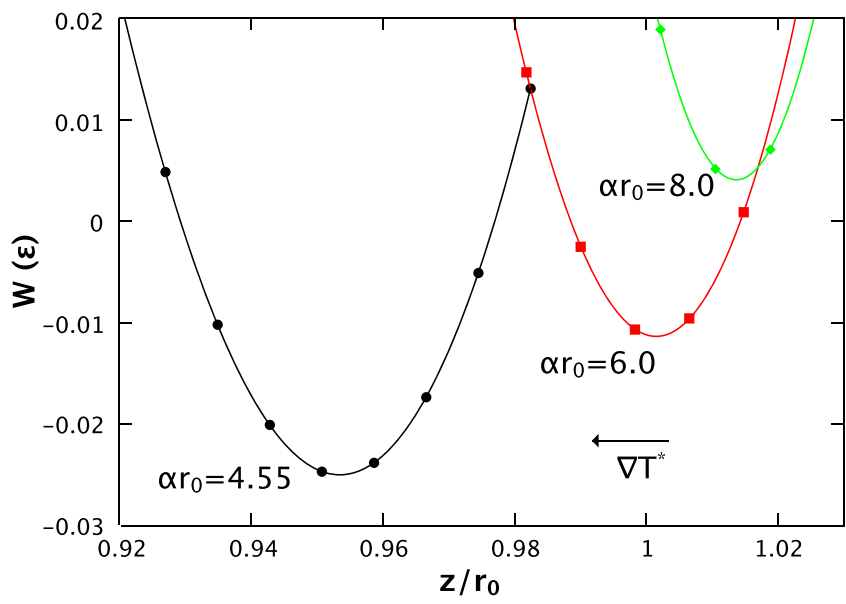

FIG. 6. Work $W(z)$ performed by the constraint force in the presence of a temperature gradient. The data are shown for the final state of the constrained atom near $z_{2}$ for $\alpha r_{0}=4.55$ (black circles), $\alpha r_{0}=6.0$ (red squares), and $\alpha r_{0}=8.0$ (green diamonds). The direction of the temperature gradient $\nabla T^{*}$ is indicated, with the arrow direction from low $T^{*}$ towards high $T^{*}$. The lines are fits to the simulation data using a third-order polynomial. 


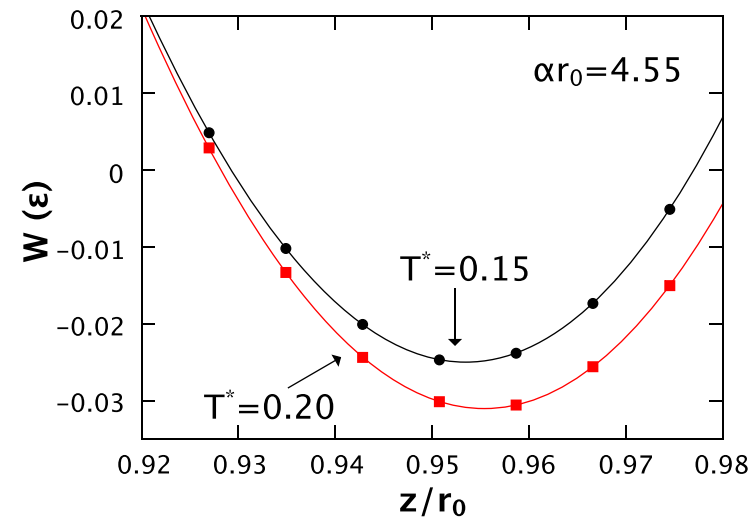

FIG. 7. Work $W(z)$ performed by the constraint force in the presence of a temperature gradient. The data are shown for the final state of the constrained atom near $z_{2}$ for $\alpha r_{0}=4.55$. The results correspond to two different averages temperatures, $T^{*}=0.15$ (black circles) and $T^{*}=0.20$ (red squares).

Using the value $\epsilon=0.5838 \mathrm{eV}$, we obtain $Q_{v}^{*}-h_{f v}$ $=-0.34 \mathrm{eV}$. This is significantly more negative that the value $Q_{v}^{*}-h_{f v}=-0.22 \mathrm{eV}$ computed at $T^{*}=0.15$ for $\alpha r_{0}=4.55$ reported in Table III.

To determine the value of $Q_{v}^{*}$, an independent calculation of $h_{f v}$ is required. To determine $h_{f v}$ and $u_{f v}$, we simulate at constant volume and constant temperature for a total of $10^{6}$ integration time steps. The system size used to find $u_{f v}$ and $h_{f v}$ was the same as in the constrained MD simulations reported above. However, in this case no atom was constrained. For each value of $\alpha r_{0}$, the system volume was held at the volume previously determined to be near the $p^{*}=0$ at $T^{*}=0.15$. To obtain $u_{f v}$ and $h_{f v}$, the time-averaged pressure and internal energy are computed for a perfect crystal with $N=3920$ atoms, and a crystal with a single vacancy and $N-1=3919$ atoms. The initial $10^{4}$ steps we used as equilibration time and not included in the time averages. We compute the vacancy formation energy from

$$
u_{f v}=\left(u_{N-1}-u_{N}\right)(N-1),
$$

where $u_{N-1}$ is the energy per atom of the system with the vacancy, $u_{N}$ is the energy per atom of the perfect crystal, and $N=3920$ is the total number of lattice sites. Likewise, the vacancy formation enthalpy is computed using

$$
h_{f v}=\left(h_{N-1}-h_{N}\right)(N-1) \text {, }
$$

where the enthalpy per atom $h_{N-1}$ of the system with a vacancy and $h_{N}$ of the perfect crystal are given by

$$
h_{N-1}=u_{N-1}+\left(\frac{p_{N-1} V}{N-1}\right) \text {, }
$$

where $p_{N-1}$ is the internal pressure of the system with a single vacancy, and

$$
h_{N}=u_{N}+\left(\frac{p_{N} V}{N}\right) \text {, }
$$

where $p_{N}$ is the pressure of the perfect crystal. While the pressure may be fairly small in each case, because the volume is the same for both the system with a vacancy and the
TABLE IV. Computed values in reduced units for the energy $u_{f v}$ and enthalpy $h_{f v}$ of vacancy formation. The reduced temperature $T^{*}=\frac{k_{B} T}{\epsilon}$ is also given. In the last column, the computed values for $Q_{v}^{*}$ are presented, using the reduced heat of transport values from Table III. The values in parentheses for $u_{f v}, h_{f v}$, and $Q_{v}^{*}$ are the values in $\mathrm{eV}$ determined for $\epsilon=0.5838 \mathrm{eV}$.

\begin{tabular}{lcccc}
\hline \hline$\alpha r_{0}$ & $T^{*}$ & $u_{f v}$ & $h_{f v}$ & $Q_{v}^{*}$ \\
\hline 4.55 & 0.15 & $8.05(4.70)$ & $5.72(3.34)$ & $5.33(3.11)$ \\
4.55 & 0.20 & $8.11(4.74)$ & $5.19(3.03)$ & $4.60(2.69)$ \\
6.0 & 0.15 & $6.87(4.01)$ & $6.78(3.96)$ & $6.55(3.82)$ \\
8.0 & 0.15 & $6.29(3.68)$ & $7.11(4.16)$ & $7.20(4.20)$ \\
\hline \hline
\end{tabular}

perfect crystal systems, the value of $p_{N}$ and $p_{N-1}$ differ. As a result, $h_{f v}$ can differ significantly from $u_{f v}$.

We include in Table IV results for each value of $\alpha r_{0}$ at $T^{*}=0.15$, and also $\alpha r_{0}=4.55$ at reduced temperature $T^{*}=0.20$. In this latter case, the volume is held at the $p=0$ volume for $\alpha r_{0}=4.55$ and $T^{*}=0.15$, and as a result the system has a significant internal pressure. The numerical error for $u_{f v}$ and $h_{f v}$ is about $\pm 0.01 \epsilon$, which is significantly smaller than the numerical error for the computed values $Q_{v}^{*}-h_{f v}$, and hence does not contribute significantly to the numerical error for $Q_{v}^{*}$. We find that $Q_{v}^{*}$ and $h_{f v}$ both tend to increase as $\alpha r_{0}$ is increased. The increase in $h_{f v}$ with $\alpha r_{0}$ is largely due to pressure effects, since we also find that $u_{f v}$ actually tends to decrease as $\alpha r_{0}$ increases. The increase of $Q_{v}^{*}$ with increasing $\alpha r_{0}$ is due in part to the computed increase in the reduced heat of transport $Q_{v}^{*}-h_{f v}$ but is in fact dominated by the strong dependence of $h_{f v}$ on $\alpha r_{0}$.

Next, we present results for the reduced heat of transport for the EAM Ni potential. In Fig. 8, the integrated work $W_{1 \rightarrow 2}$ in the presence of a temperature gradient at an average temperature $T=450 \mathrm{~K}$ for the Ni EAM potentials is shown. The integrated work to displace the atom from $z_{1}$ to $z_{2}$ is $-0.004 \pm 0.002 \mathrm{eV}$. For the imposed heat current, the temperature difference $\Delta T=T_{1}-T_{2}=20.8 \pm 2 \mathrm{~K}$. Using these values in Eq. (1), the value $Q_{v}^{*}-h_{f v}=-0.08 \pm 0.04 \mathrm{eV}$ is obtained. This value is clearly negative, but of a rather small magnitude in comparison to the values obtained with the

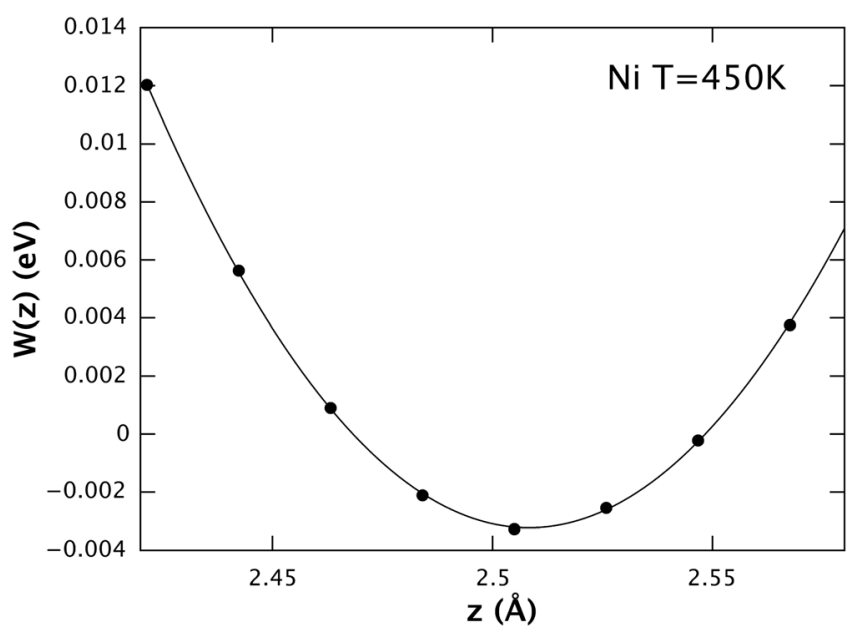

FIG. 8. Work $W(z)$ performed by the constraint force in the presence of a temperature gradient for the Ni EAM potential with $T=450 \mathrm{~K}$. The data are shown for the final state of the constrained atom near $z_{2}$. 
Morse potentials. The activation entropy was also computed during the first $2 \times 10^{5} \mathrm{MD}$ steps and found to be $\Delta S_{a}=(2.2 \pm 0.2) k_{B}$. This value is positive, as are the values for the Morse potentials with $\alpha r_{0}=4.55$ and $\alpha r_{0}=6.0$, but is of an even larger magnitude. The vacancy heat of formation was computed to be $h_{f v}=1.19 \mathrm{eV}$, resulting in a value $Q_{v}^{*}=1.11 \mathrm{eV}$. As expected, the more realistic value of $h_{f v}$ obtained when using an EAM potential leads to a more reasonable value for $Q_{v}^{*}$ in comparison to pair potentials, which are known to greatly overestimate $h_{f v}$.

\section{ANALYSIS}

In this paper, we report extensive simulation results relevant to thermodiffusion of vacancies in a single-component system. In contrast to previous studies, the objective was to gain some understanding of what controls the relevant transport parameters $Q_{v}^{*}-h_{f v}$ and $Q_{v}^{*}$ by systematically varying the potential parameters in a simple model. We find, as previously reported in Ref. 1 , that the value of $Q_{v}^{*}$ is close to the vacancy formation enthalpy $h_{f v}$. This prediction is in agreement with simulations reported in Refs. 2 and 24 for the heat of transport of hydrogen in a Pd lattice. We think that this is an important insight that likely is characteristic of most thermodiffusion problems. This point remains to be verified by exploring other materials systems.

The reduced heat of transport $Q_{v}^{*}-h_{f v}$ was found to depend systematically on the value of $\alpha r_{0}$ used in the Morse potential. More insight was obtained from the observation that the activation entropy $\Delta S_{a}$ also depended systematically on $\alpha r_{0}$. The results here suggest an important correlation between $Q_{v}^{*}-h_{f v}$ and $\Delta S_{a}$ that can enable improved physical understanding.

It can be readily understood why $Q_{v}^{*}-h_{f v}$ might be correlated to $\Delta S_{a}$. In particular, the activation entropy is related to differences between the work required to drive an atom to the transition state and the change in internal energy when the system is maintained at constant and uniform temperature $T$. If the vacancy is driven through the transition state from one free-energy minimum to another, the net change in energy, entropy, and free energy is zero. When there is a temperature gradient present, $T$ is not uniform, and as a result the work $W_{1 \rightarrow 2}$ required to drive the diffusing atom is nonzero primarily due to entropic effects along the diffusion path. The general trend we predict here is that $Q_{v}^{*}-h_{f v}$ tends to be negative when $\Delta S_{a}$ is large and positive. When $\Delta S_{a}$ trends towards zero, the magnitude of $Q_{v}^{*}-h_{f v}$ becomes smaller. As $\Delta S_{a}$ becomes large and negative, $Q_{v}^{*}-h_{f v}$ can become positive.

Agreement between theoretical predictions and experimental results is still not completely realized for the case of single-component materials. In Table V, we compare to previous experimental and computational results. For temperatures near $T=1100 \mathrm{~K}$, the simulations reported here and in Refs. 8 and 9 agree qualitatively with experiment for $\mathrm{Au} .^{21-23}$ In particular, for the case of $\mathrm{Au}$, the value of $Q_{v}^{*}-h_{f v}$ is found to be negative in both experiment and simulation. However, the magnitude of the values predicted by simulation is significantly different from what is found in
TABLE V. Comparison between simulation and experimental results for $Q_{v}^{*}-h_{f v}$ and $Q_{v}$. Experimental results are from Refs. 17, 18, and 23-25 for $\mathrm{Au}$ and $\mathrm{Cu}$. Simulation results from Ref. 8 most closely correspond to $\mathrm{Au}$, as do the simulation results reported in this paper for $\alpha r_{0}=4.55$. The values for the temperatures used in the experiment are comparable to the average values reported.

\begin{tabular}{llrrrr}
\hline \hline & & \multicolumn{4}{c}{$Q_{v}^{*}-h_{f v}$} \\
System & Exp./Sim. & $T(\mathrm{~K})$ & $(\mathrm{eV})$ & $h_{f v}$ & $Q_{v}^{*}(\mathrm{eV})$ \\
\hline $\mathrm{Au}($ Ref. 8) & Sim. & 730 & -3.9 & 3.31 & -0.59 \\
$\mathrm{Au}($ Ref. 8) & Sim. & 1100 & -2.0 & 3.31 & 1.31 \\
$\mathrm{Au}($ Ref. 8) & Sim. & 1440 & -1.2 & 3.31 & 2.11 \\
$\mathrm{Au}($ Refs. 17, 18, and 22) & Exp. & $\sim 1100$ & -0.59 & 0.98 & 0.39 \\
$\mathrm{Au}($ Refs. 17, 18, and 21) & Exp. & $\sim 1100$ & -0.68 & 0.98 & 0.30 \\
$\mathrm{Au}($ Refs. 17, 18, and 23) & Exp. & $\sim 1100$ & -0.80 & 0.98 & 0.18 \\
$\mathrm{Cu}($ Refs. 18 and 23) & Exp. & $\sim 1100$ & -1.37 & 1.17 & -0.20 \\
$\mathrm{Au} \alpha r_{0}=4.55$ (This work) & Sim. & 1016 & -0.22 & 3.34 & 3.11 \\
$\mathrm{Au} \alpha r_{0}=4.55$ (This work) & Sim. & 1354 & -0.34 & 3.03 & 2.69 \\
$\mathrm{Ni}$ EAM (This work) & Sim. & 450 & -0.08 & 1.19 & 1.11 \\
\hline \hline
\end{tabular}

experiment. The calculations reported in Refs. 8 and 9 predict the magnitude of $Q_{v}^{*}-h_{f v}$ at least twice that found in experiment for $\mathrm{Au}$. In the present calculations, we find that $Q_{v}^{*}-h_{f v}$ is smaller than the experimental results for Au by at least a factor of two.

By computing $h_{f v}$, we determined values for $Q_{v}^{*}$ that also can be compared with experiment. In the case of the results reported in Refs. 8 and 9, we use the computed value for $h_{f v}$ at $T=0$ using the Cherns potential. ${ }^{12}$ For the experimental results, we use the reported values for $Q_{v}^{*}-h_{f v}{ }^{21-23}$ and $h_{f v}{ }^{17,18} \mathrm{We}$ find that for the case of Au at $T \sim 1100 \mathrm{~K}$, the results in Table $\mathrm{V}$ all predict a positive value for $Q_{v}^{*}$, and hence experiment and simulation are at least in qualitative agreement. However, the simulations here and in Refs. 8 and 9 predict values substantially larger than what is seen in experiment. The results in Table V suggest perhaps the most significant source of error in predicting $Q_{v}^{*}$ is due to the inability of both the Cherns and Morse potentials to accurately reproduce the value of $h_{f v}$. In fact, the results in Table V show that the Morse and Cherns potentials all systematically overestimate the value of $h_{f v}$.

It seems very likely, therefore, that the most important source of error in the present calculations of $Q_{v}^{*}$ lies in the fact that no simple pair potential can accurately predict $h_{f v}$. However, we also note that the simulations reported here for $\alpha r_{0}=4.55$ and those in Refs. 8 and 9 do not accurately predict the enthalpy of vacancy migration $h_{m v}$. As we have seen here, the details of the energetics through the transition state are closely related to the predicted value of $Q_{v}^{*}-h_{f v}$, and so it seems likely that the predictions reported here and in Refs. 8 and 9 might be significantly in error when compared to experimental results for Au. For example, the results in Table III for $\alpha r_{0}=4.55$ show that the energy of activation for vacancy migration is $\Delta U_{a}=1.84 \mathrm{eV}$. The value of $\Delta U_{a}$ should be quite close to $h_{m v}$. In Ref. 8 and 9, the value of $h_{m v}$ is significantly larger, in particular $h_{m v}=2.64 \mathrm{eV}$. In contrast to these calculated values, the experimental value of $h_{m v}=0.83 \mathrm{eV}$ (Ref. 23) is significantly smaller. Thus, the details of the energy profiles along the diffusion path, and 
most likely the entropy profile as well, are not accurately predicted by the Morse or Cherns potentials.

Results obtained using the EAM potential for Ni demonstrate a value for $Q_{v}^{*}$ that is significantly smaller in magnitude than those reported for the Morse potentials primarily due to the smaller value of $h_{f v}$. It is well known that EAM potentials are able to reproduce accurately the formation enthalpy of vacancies due to the many-body effects incorporated in the formalism.

Finally, we comment on the negative value of $Q_{v}^{*}$ reported in experiment for $\mathrm{Cu}^{18,21}$ We note that the measured value of $Q_{v}^{*}-h_{f v}$ is negative, but of the same general magnitude as those predicted using simulation here and in Refs. 8 and 9. Therefore, it is entirely reasonable that a pairtype potential could predict the observed value for $Q_{v}^{*}-h_{f v}$ in $\mathrm{Cu}$. However, given the inability of simple pair potentials to predict $h_{f v}$, we expect that pair potentials will always predict a positive value for $Q_{v}^{*}$. While a positive value for $Q_{v}^{*}$ was found using the EAM model for $\mathrm{Ni}$, it may be possible for an EAM potential to result in a negative value for $Q_{v}^{*}$. We also note that, based on the insight obtained in this paper, the rather large negative value for $Q_{v}^{*}-h_{f v}$ measured for $\mathrm{Cu}$ suggests that $\Delta S_{a}$ should be fairly large and positive. We do not know of any existing calculations that predict values for $\Delta S_{a}$ in bulk $\mathrm{Cu}$ and $\mathrm{Au}$ using either EAM or densityfunctional theory calculations. However, it would be interesting to establish whether this correlation between $\Delta S_{a}$ and $Q_{v}^{*}-h_{f v}$ can be verified for the two cases of $\mathrm{Cu}$ and $\mathrm{Au}$.

The value of $Q_{v}^{*}-h_{f v}$ and $Q_{v}^{*}$ were found to depend on system temperature. Temperature dependence was also predicted in Refs. 8 and 9. In experiments, there have been few systematic studies of temperature dependence, and so the situation from experiment is not totally clear. There are exceptions, however. For example, systematic dependence in ionic crystals has been known for some time (see Ref. 19, for a review). In the case of metals, a systematic study in the case of Pt has been reported in Ref. 20, where the heat of transport for vacancies was found to be negative with a magnitude that increases with temperature. In the calculations reported here, we find that as $T^{*}$ increases, $Q_{v}^{*}-h_{f v}$ becomes more negative for the case studied with $\alpha r_{0}=4.55$. Consequently, $Q_{v}^{*}$, while still positive in the simulations reported here, tends to decrease in magnitude as $T^{*}$ increases. In the simulations reported here, internal stress may also play a role, but this aspect was not investigated. In particular, the system volume was kept the same for $T^{*}=0.15$ and $T^{*}=0.20$ with $\alpha r_{0}=4.55$, resulting in very different internal stress. It is therefore not possible to isolate dependence on temperature and stress. Nevertheless, temperature dependence has clearly been demonstrated, which is consistent with the observation that $Q_{v}^{*}-h_{f v}$ is strongly correlated with the sign and magnitude of the activation entropy $\Delta S_{a}$.

\section{SUMMARY AND CONCLUSIONS}

In summary, using pair potentials and EAM potentials, the constrained-dynamics method was used to compute the heat of transport in single-component fcc crystals. Simulations included three different Morse potentials and an
EAM potential for Ni. The results were consistent with recently published Green-Kubo simulations for hydrogen thermodiffusion in a Pd lattice. Specifically, the heat of transport is comparable to the partial enthalpy of the diffusing species. In the results here, this leads to relatively small values for $Q_{v}^{*}-h_{f v}$. In addition, the results here were qualitatively similar to the previous study of thermodiffusion in Lennard-Jones crystals. ${ }^{1}$

The results suggest a possibly important trend; namely, that $Q_{v}^{*}-h_{f v}$ is strongly correlated with $\Delta S_{a}$, indicating that entropic effects along the diffusion path control thermodiffusion. Dependence on system temperature was also observed, which could also be attributed to entropic effects. However, while the correlation is clearly established, it is not clear if there is any physical significance underlying the correlation, nor whether or not this is a general result.

The lack of agreement between theory and experiment is still an important shortcoming of this work and those published by other authors. One clear problem is that there is generally very little data for single-component systems, and published experimental results, for example, for $\mathrm{Au}$ and $\mathrm{Cu}$, have rather large error bars. For predictions of $Q_{v}^{*}$, we have shown that pair potentials are generally unsuitable because they fail to accurately predict $h_{f v}$. The study of binary alloys is another important direction due to the greater relevance to applications and the existence of experimental data still not explained theoretically. For example, the methodology developed in Ref. 1 and used here should be applied to dilute solid solutions, with the results compared to experiment.

In Ref. 24, it was demonstrated that the thermodiffusion of hydrogen atoms in a Pd lattice could be understood by computing individual contributions to the integrated correlation functions. In particular, it was found that the hopping hydrogen atoms have slightly higher kinetic and potential energies than average. In addition, in Ref. 24, we showed that the contribution due to the virial terms in the heat current was slightly less than what might be expected based on the average partial pressure of hydrogen. These results suggest that essentially the reduced heat of transport is the difference between the partial enthalpy of a hydrogen atom during a hop and the average enthalpy of a hydrogen atom. Specifically, hydrogen atoms that undergo a hopping event have a different kinetic energy, potential energy, and different contribution to the stress tensor than an average hydrogen atom. Assuming this is a general result, it should be possible to apply this picture to the thermodiffusion of vacancies. In particular, the results here indicate that $Q_{v}^{*}-h_{f v}$ is relatively small. We propose that differences between $Q_{v}^{*}$ and $h_{f v}$ are due to the difference between the partial enthalpy of a vacancy undergoing a hopping event and a vacancy at a potential minimum in the lattice.

Finally, we comment on the relationship between the constrained-dynamics method and previous approaches, including Green-Kubo and the method derived from the original work due to Gillan. ${ }^{3-5}$ We think that the constraineddynamics method as presented can be compared directly to experiment and Green-Kubo simulations. As already noted, essentially exact agreement has been found between constrained-dynamics and Green-Kubo for thermodiffusion 
in the Pd:H system. ${ }^{2}$ As for comparison with the method originally due to Gillan, the significant disagreement reported here requires some additional comment. In the Gillan approach, the dynamics of atomic hops is explicitly computed, in contrast with the constrained-dynamics approach. In simulating the dynamics of atom hops, a large heat flux was observed that makes a dominant contribution to the computed value of the heat of transport. ${ }^{6-9}$ This effect is not present in the constrained-dynamics method. Some of us discuss this further elsewhere. ${ }^{25}$

\section{ACKNOWLEDGMENTS}

This work was supported by a grant from the National Science Foundation (Award No. 1106219). We also acknowledge support from the STOKES computing cluster at UCF housed at the Institute for Simulation and Training.

\section{APPENDIX: CONSTRAINED DYNAMICS AND THE SECOND LAW OF THERMODYNAMICS}

Here, we describe the origin of Eq. (1) in terms of a series of thermodynamic processes, as we did in Ref. 1, with an emphasis on the role of entropy production. We also note that no violation of the second law of thermodynamics is implied.

We first envision that a vacancy has hopped from $z_{2}$ to $z_{1}$ irreversibly with entropy generation $\Delta_{i} S_{\text {hop }}>0$ given by

$$
\Delta_{i} S_{h o p}=\left(Q_{v}^{*}-h_{f v}\right) \frac{T_{2}-T_{1}}{T^{2}} .
$$

Rather than viewing this process in terms of the displacement of the vacancy, it can equivalently be viewed as the hopping of an atom from $z_{1}$ to $z_{2}$. After the irreversible hop occurs, we next envision the process of returning the system to its original state by applying an external force to displace the vacancy from $z_{1}$ to $z_{2}$, or equivalently displacing an atom from $z_{2}$ to $z_{1}$. Hence, we view the process of returning the system to its original state as using work $W_{2 \rightarrow 1}$ done on the system by the external force to move an atom from $z_{2}$ to $z_{1}$. In this process, the atom is displaced infinitely slowly and the heat flux $J_{q}$ is held constant. If this process is done infinitely slowly, then it can be done reversibly (i.e., without generating any entropy in the system). However, this process increases the internal energy of the system by an amount $W_{2 \rightarrow 1}$, and in order to return the system to its original state this energy must be removed from the system to the external environment. This changes the entropy of the system by an amount $-W_{2 \rightarrow 1} / T$. After returning the vacancy to its original position and releasing heat $W_{2 \rightarrow 1}$ to the environment, the entropy of the system should be the same as it was before the irreversible vacancy hop from $z_{2}$ to $z_{1}$. Hence, we have for the overall change in entropy of the system

$$
-W_{2 \rightarrow 1} / T+Q_{v}^{* \prime}\left(\frac{T_{2}-T_{1}}{T^{2}}\right)=0 .
$$

From this expression, Eq. (1) can be derived.
The process outlined above does not violate the laws of thermodynamics. Specifically, after an irreversible process, in this case a vacancy hop in the presence of a temperature gradient, the system can be returned to its original state only by increasing the entropy of the environment. In the process outlined above, the reversible work due to the external constraint force returns the system to its original state, but only with the release of heat $W_{2 \rightarrow 1}$ to the environment. For example, if the environment is also at temperature $T$, then the increase in the entropy of the environment is $W_{2 \rightarrow 1} / T$. Therefore, after the process outlined above, the entropy of the system is unchanged, but the entropy of the environment has increased.

${ }^{1}$ Z. McDargh and P. K. Schelling, "Molecular-dynamics approach for determining the vacancy heat of transport," Comput. Mater. Sci. 50, 2363 (2011). ${ }^{2} \mathrm{P}$. K. Schelling and T. Le, "Computational methodology for analysis of the Soret effect in crystals: Application to hydrogen in palladium," J. Appl. Phys. 112, 083516 (2012).

${ }^{3}$ M. J. Gillan, "The heat of transport in solids: A new theoretical approach," J. Phys. C: Solid State Phys. 10, 1641 (1977).

${ }^{4}$ M. J. Gillan, "Heat of transport in solids: II. Harmonic theory for a vacancy in the simple cubic lattice," J. Phys. C: Solid State Phys. 10, 3051 (1977).

${ }^{5}$ M. J. Gillan and M. W. Finnis, "Heat of transport in solids: III. Computer simulation of a vacancy in an fcc crystal," J. Phys. C: Solid State Phys. 11, 4469 (1978).

${ }^{6}$ C. Jones, P. J. Grout, and A. B. Lidiard, "The heat of transport of vacancies in solid argon,” Philos. Mag. Lett. 74, 217 (1996).

${ }^{7}$ C. Jones, P. J. Grout, and A. B. Lidiard, "The heat of transport of vacancies in model fcc solids," Philos. Mag. A 79, 2051 (1999).

${ }^{8} \mathrm{P}$. J. Grout and A. B. Lidiard, "Computation of heats of transport in crystalline solids: II,” J. Phys. Condens. Matter 20, 425201 (2008).

${ }^{9}$ K. A. M. Dickens, P. J. Grout, and A. B. Lidiard, "Computation of heats of transport of vacancies in model crystalline solids: III," J. Phys. Condens. Matter 23, 265401 (2011).

${ }^{10}$ R. E. Howard and A. B. Lidiard, "Matter transport in solids," Rep. Prog. Phys. 27, 161 (1964).

${ }^{11}$ G. Galliero and S. Volz, "Thermodiffusion in model nanofluids by molecular dynamics simulations," J. Chem. Phys. 128, 064505 (2008).

${ }^{12}$ D. Cherns, M. W. Finnis, and M. D. Matthews, Philos. Mag. 35, 693 (1977).

${ }^{13} \mathrm{Y}$. Mishin, "Atomistic modeling of the $\gamma$ and $\gamma^{\prime}$ phases of the Ni-Al system," Acta Mater. 52, 1451 (2004).

${ }^{14}$ G. P. Purja Pun and Y. Mishin, "Development of an interatomic potential for the Ni-Al system," Philos. Mag. 89, 3245 (2009).

${ }^{15} \mathrm{P}$. Jund and R. Jullien, "Molecular-dynamics calculation of the thermal conductivity of vitreous silica," Phys. Rev. B 59, 13707 (1999).

${ }^{16}$ J. H. Harding, "Computer simulation of defects in ionic solids," Rep. Prog. Phys. 53, 1403 (1990).

${ }^{17}$ R. O. Simmons and R. W. Balluffi, "Measurement of the equilibrium concentration of lattice vacancies in gold," Phys. Rev. 125, 862 (1962).

${ }^{18}$ R. O. Simmons, J. S. Koehler, and R. W. Ballufi, in Radiation Damage in Solids (International Atomic Energy Agency, Vienna, 1962), Vol. 1.

${ }^{19}$ J. Janek, C. Korte, and A. B. Lidiard, "Thermodiffusion in ionic solidsModel experiments and theory," in Thermal Nonequilibrium Phenomena in Fluid Mixtures, Springer Lecture Notes in Physics, Vol. 584, edited by W. Kohler and S. Wiegand (Springer, 2002).

${ }^{20}$ S. C. Ho, Th. Hehenkamp, and H. B. Huntingdon, "Thermal diffusion in platinum," J. Phys. Chem. Solids 26, 251 (1965).

${ }^{21}$ C. J. Meechan and G. W. Lehman, "Diffusion of $\mathrm{Au}$ and $\mathrm{Cu}$ in a temperature gradient," J. Appl. Phys. 33, 634 (1962).

${ }^{22}$ D. Jaffe and P. G. Shewmon, Acta Metall. 12, 515 (1964).

${ }^{23}$ W. Mock, "Thermomigration of $\mathrm{Au}^{195}$ and $\mathrm{Sb}^{125}$ in gold," Phys. Rev. 179, 663 (1969).

${ }^{24}$ W. C. Tucker, L. Shokeen, and P. K. Schelling, "Atomic-scale simulation of the thermodiffusion of hydrogen in palladium," J. Appl. Phys. 114, 063509 (2013).

${ }^{25}$ W. C. Tucker and P. K. Schelling, "Analysis of simulation methodology for calculation of the heat of transport for vacancy thermodiffusion," (in press). 